\title{
STUDYING DESIGN ATTRIBUTES OF VIRTUAL CHARACTERS TO SUPPORT STUDENTS' PERCEIVED EXPERIENCES IN VIRTUAL REALITY LECTURES
}

\author{
Brittany Garcia ${ }^{1}$, Soo Chun ${ }^{1}$, Caleb Kicklighter ${ }^{1}$, Bin $\mathrm{Mai}^{2}$, Marco Palma ${ }^{3}$ \\ and Jinsil Hwaryoung Seo ${ }^{1}$ \\ ${ }^{1}$ Department of Visualization Texas A\&M University, College Station, TX, USA \\ ${ }^{2}$ Department of Educational Administration \& Human Resource Development, College Station, TX, USA \\ ${ }^{3}$ Department of Agriculture and Economics, College Station, TX, USA
}

\begin{abstract}
Virtual reality recently expanded to collaborative and social networking environments where users interact with virtual characters (VCs) of various appearances and behaviors, often impacting the quality of experience. In this paper, we present preliminary survey results of perceptions about characteristics of VCs and a user study of a VR lecture app with different styles of virtual instructors. VCs were collected from existing collaborative and social VR applications and categorized by body types and design style. Survey results coupled with prior works guided the development of design styles, which we briefly present. In the user study, 12 users experienced two different VR lectures with randomly assigned character styles. Results show participants found the stylized VC familiar and human while the realistic VC was engaging and trustworthy. Results also show feelings of learning more about the lecture topic with the stylized VC.
\end{abstract}

\section{KEYWORDS}

Virtual Reality, Virtual Character Design, 3D Avatar Design, Perception, Virtual Reality Lecture, Immersive Learning

\section{INTRODUCTION}

Virtual Reality (VR) environments support a wide range of activities like activities in real environments (Sung, Moon, and Lin, 2011). They are rapidly becoming popular in training, education, and entertainment (Yee, Bailenson, and Rickertsen, 2007), due to VR's interactive and embodied experiences which cannot be easily accessible or possible in the real world (Erolin, Reid, and McDougall, 2019). In addition, the recent expansion of VR technology during the COVID-19 pandemic allows users to meet and work together in a collaborative or social VR environment. In these virtual environments, users often interact with virtual characters (VCs) or avatars: a user plays with another player's avatar in a VR game; a user follows an instruction from a $\mathrm{VC}$ in a training application. Users select avatar designs from a list of options or design their own avatar with detailed parts. Different brands or applications provide unique styles on VCs' appearances and behaviors.

Research shows that visual styles of VCs have subsequent impacts on performance in virtual environments (Erolin, Reid, and McDougall, 2019). Yee and Bailenson (2007) found that appearance cues of a $\mathrm{VC}$ led users to experience attitudinal and behavioral changes in a manner stereotypically consistent with these cues (Yee and Bailenson, 2007). In educational VR applications, the appearance cues of VCs for instructors, experts, and colleagues are important to create positive impacts on students' learning and learning related experiences. However, there is no strong body of research guiding the design of VCs for VR learning environments. Therefore, we address the following research question in this paper: How do different visual types of VCs impact users' perception and support learning?In this paper, we present preliminary survey results of perceptions on characteristics of VCs, which were collected from existing collaborative and social VR applications and categorized by body types and design style. Then we present a user study of a VR lecture app that we created. This application includes different design styles of virtual instructors. Finally, we discuss meaningful insights of designing virtual instructor characters for educational VR applications. 


\section{BACKGROUND}

\subsection{Perception of Virtual Characters}

VCs exist within computer simulations and have a perceptible digital character whose behavior is executed by self, others or generated by an intelligent system (Yee and Bailenson, 2007). The visual style of VCs varies; appearance can be influenced by genre, target-audience, or cultural differences. Various design features of VCs' appearances impact participants' emotions, behaviors, interactions and so on. Steptoe et al. (2010) assessed the impact of the addition of realistic eye motion in avatar-mediated communication and found realistic eye movement increases participant accuracy in detecting truth and deception when interacting with virtual avatars (Steptoe et al, 2010).

The domain of VR aims to design characters to foster immersion in the virtual world. Sometimes highly realistic characters create negative reactions and break immersion. This was first observed in the 1970s by Masahiro Mori, a roboticist who noticed humans' negative response to robots or prosthetics that are not quite human-like (Mori, MacDorman, and Kageki, 2012). Mori called this the uncanny valley, which is a serious challenge for designers when creating VCs. Guidelines to avoid the uncanny valley to enhance the quality of experience are needed to design successful products.

\subsection{VCs in Educational VR}

VCs have been used to deliver educational content as part of a vision that virtual instructors can advantageously influence learning (Krämer and Bente, 2010). These characters have an identifiable body and communicate using voice, gesture, or facial expression for instructional purposes. They embody instructional objectives and goals (Paiva and Machado, 1998; Johnson, Rickel, and Lester, 2000) to support face-to-face interaction in learning environments and have been extensively applied to Virtual Learning Environments that utilize immersive head-mounted displays (HMDs) or virtual reality rooms (Dede, 2009).

Prior research suggests the presence of a human-like animated character can have a positive influence on students' learning experience. For example, people experience more positive emotions when interacting with a VC that gives positive feedback (Pour et al, 2010). Other work has found that people who viewed a human-voiced character with facial expression and body movements performed better on a test of knowledge transfer compared to either a nonmoving character or no character (Mayer and DaPra, 2012; Wagner, Billinghurst, and Schmalstieg, 2006). Further, it has been proposed that in terms of VCs, it is not the presence of the VC itself, but its behavior and the instructional method embedded in its use that impacts positively on learning (Krämer and Bente, 2010). Therefore, the design of VCs focusing on appearance and behavior matters in educational VR environments.

\section{PRELIMINARY STUDY IN VC PERCEPTION}

To obtain users' general perception of VCs in virtual environments, we developed a survey by collecting 89 VC images with different body types (e.g., full body, half body, torso), styles (e.g., stylized, realistic, non-human) and skin tone (e.g., light, medium, dark) from existing collaborative and social VR applications. Researchers collected images from the following applications: Vspatial (Platt and Tucker, n.d.), Mozillahub (Mozilla Mixed Reality Team, 2018), Rumii (Doghead Simulations, 2017), Spatial (Agarwala, A. and Lee, J. 2016), AltSpace (Microsoft, 2015), Engage (Immersive VR Education Ltd, 2016), VIVE Sync (HTC Vive, 2018), Virbela (Howland, 2012) and VrChat (VRChat Inc., 2014). Researchers took the following steps in selecting the VC images: i.) select VC gender; ii.) select the skin tone, if applicable; iii.) select business casual clothing option for $\mathrm{VC}$ if this option was not available researchers went with attire the application automatically generated for the $\mathrm{VC}$; and iv.) researchers took a screenshot of the VC. In the survey a total of 10 representative $\mathrm{VC}$ images were presented to participants (Figure 1). These 10 avatars were chosen based on a discrete choice experiment (DCE) which we also used to determine participants preferences. In a DCE preference for a product or service, in our case VCs, are decomposed as preferences for the attributes of a 
VC. In essence, a product is viewed as a bundle of attributes and we evaluate the customer, or participant, response to each attribute. The first step in designing the experiment is to select the most relevant attributes and attribute levels for a VC (e.g., gender, body type, skin tone and style). Given the number of attributes and attribute levels, there are a total of 54 possible combinations of attributes. Since it is not feasible to ask respondents to evaluate all possible combinations, we used a fractional factorial design using the \%ChoiceEff macro in SAS 9.4 with a modified Fedorov algorithm to optimize the VC combinations (Kuhfeld, 2013). In particular, the design is optimized for orthogonality (independence of the attributes) and balance (the attributes appearing the same number of times). The final design consisted of 9 choice sets of two VC alternatives with a D-efficiency measure of $95.68 \%$. Researchers chose ten combinations of the VCs based on the SAS results. Researchers tested this first draft of the survey, asking about $20 \mathrm{VCs}$, with six individuals and found that the participants found the survey to be too long lasting 1.5 to 2 hours, therefore researchers chose ten VCs of the for the final survey (Figure 1).
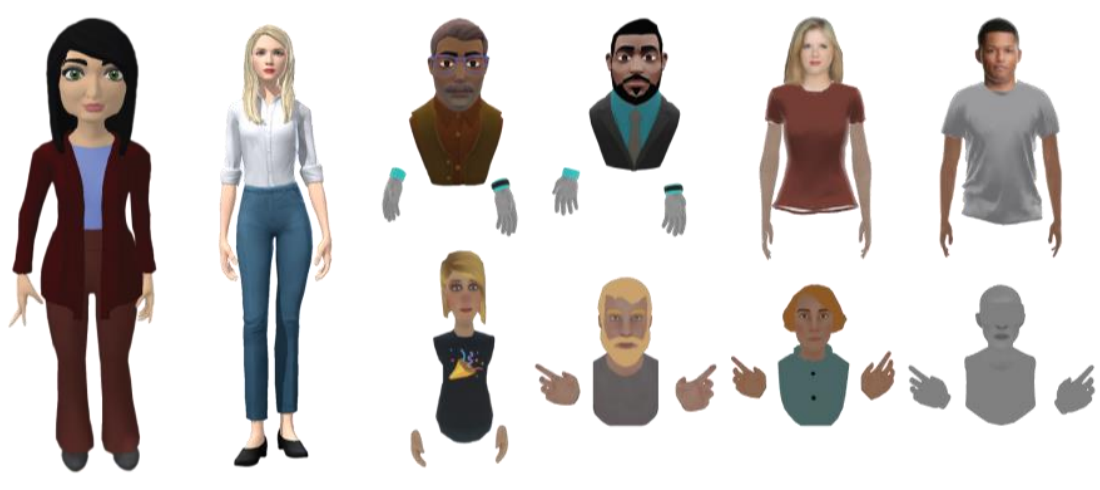

Figure 1. Representative virtual characters for survey

Likert scales were used to rate characters on familiarity (Hiroi, Ito, and Nakano, 2009), engagement (IJsselsteijn, de Kort, and Poels 2013), trust (Chae, Lee, and Seo, 2016) and humanness (Ho and MacDorman, 2010), which are critical perceptive aspects when users interact with VCs. We asked participants to imagine the style (e.g., robot, stylized, realistic, other, unsure) they preferred for a VC instructor. Researchers were interested in investigating preference, to do so a discrete choice experiment (DCE) was implemented to assess participant's preferences for VCs. Participants choose the option they prefer, and their choice provides a measure of their preference. This experimental design has two main advantages. First, it provides more realistic VC environments by providing actual VCs instead of asking participants to simply rate their liking for individual design features. Second, it is easier and more intuitive for participants to choose the VC character that they would prefer in an educational context. The survey was distributed via university bulk email, word of mouth and social media.

\subsection{Results}

A total of 105 responses were collected, 36 males, 68 females and 1 prefer not to say aged 18-24 (54.29\%), $25-34(19.05 \%), 35-44(11.43 \%), 45-54(8.57 \%), 55-64(5.71 \%)$ and $65+(.95 \%) .64 .76 \%$ of participants reported experience with VR using head mounted displays and mobile VR. $39.81 \%$ reported experience with VCs in VR with the most common style being stylistic in gaming applications. Participants reported preferences for stylized and realistic VCs as educational instructors. Analysis of variance results are reported in the sections below along with the results for a mixed logit model to determine participants' VC preference.

\subsubsection{Familiarity}

An independent one-way ANOVA showed a significant effect of VC body (full body, half body and torso) on familiarity $(\mathrm{F}(2,1057)=16.78, \mathrm{p}<.001)$. Post hoc testing revealed full bodies resulted in greater familiarity than half bodies $(\mathrm{p}<.001)$. There were also significant differences in familiarity between half and torso bodies $(\mathrm{p}<.001)$, with torso being more familiar. There were no significant differences in familiarity between full and torso bodies $(\mathrm{p}=.588)$. An independent one-way ANOVA showed a significant effect of VC style 
(stylized, realistic, and non-human) on familiarity $(\mathrm{F}(2,1056)=19.231, \mathrm{p}<.001)$. Post hoc testing revealed that stylized VCs were significantly more familiar than realistic style $(\mathrm{p}<.001)$ and non-human styles were significantly more familiar than realistic styles $(\mathrm{p}=.025)$. There were no significant differences in familiarity between stylized and non-human styles ( $\mathrm{p}=.515)$.

\subsubsection{Engagement}

An independent one-way ANOVA showed a significant effect of body type on engagement $(F(2,1057)$ $=6.120, \mathrm{p}=.002)$. Post hoc testing revealed torso bodies resulted in greater engagement than half bodies $(p=.002)$. There were no significant differences in engagement between full and half bodies $(p=.089)$ nor between full and torso bodies $(\mathrm{p}=.743)$. An independent one-way ANOVA showed a significant effect of style on engagement $(\mathrm{F}(2,1057)=10.776, \mathrm{p}<.001)$. Post hoc testing revealed stylized VCs were significantly more engaging than realistic styles $(\mathrm{p}<.001)$. There were no significant differences in engagement between stylized and non-human styles $(\mathrm{p}=.517)$ nor realistic and non-human styles $(\mathrm{p}=.202)$.

\subsubsection{Trust}

An independent one-way ANOVA showed a significant effect of body type on trust $(\mathrm{F}(2,1057)=6.834$, $\mathrm{p}=.001$ ). Post hoc testing revealed torso bodies were significantly more trustworthy than half bodies $(\mathrm{p}<.001)$. There were no significant differences in trust between full and half bodies $(\mathrm{p}=.150)$ nor full and torso bodies $(\mathrm{p}=.458)$. An independent one-way ANOVA showed a significant effect of style on trust $(\mathrm{F}(2,1057)=9.554, \mathrm{p}<.001)$. Post hoc testing revealed stylized VCs were significantly more trustworthy than realistic style $(\mathrm{p}<.001)$. There were no significant differences in trust between stylized and non-human styles $(\mathrm{p}=.750)$ nor realistic and non-human styles $(\mathrm{p}=.139)$.

\subsubsection{Humanness}

An independent one-way ANOVA showed a significant effect of body type on familiarity $(\mathrm{F}(2,1057)$ $=14.072, \mathrm{p}<.001)$. Post hoc testing revealed full bodies were significantly more human than half bodies $(\mathrm{p}=001)$. There were also significant differences in humanness between half and torso bodies $(\mathrm{p}<.001)$, with torso being greater. There were no significant differences in humanness between full and torso bodies $(\mathrm{p}=.742)$. An independent one-way ANOVA showed a significant effect of style on humanness $(\mathrm{F}(2,1057)$ $=11.383, \mathrm{p}<.001)$. Post hoc testing revealed stylized VCs were significantly more human than realistic style $(\mathrm{p}<.001)$ and non-human styles were more human than realistic $(\mathrm{p}=.002)$. There were no significant differences in humanness between stylized and non-human style $(\mathrm{p}=.708)$.

\subsubsection{Mixed Logit Model}

Since in our experimental design participants are choosing between two products (VCs), we estimate a mixed logit model. Authors will first explain the purpose of a mixed logit model and then report the model's results. A mixed logit model allows for the estimation of mean preference parameters and standard deviations to provide measures of preference heterogeneity. The mean parameters represent the average preference of the entire sample. A positive parameter indicates that the specified attribute increases the probability of the VC being chosen. Hence, a positive coefficient is interpreted as a most preferred design feature. Similarly, a negative mean parameter indicates a lower likelihood of choosing the $\mathrm{VC}$ for that attribute and therefore a lower preference for that attribute. A statistically significant Standard Deviation parameter provides indication of preference heterogeneity. That is that some respondents may prefer an attribute while other respondents dislike it. The MEAN parameters show the likelihood of choosing an avatar with those characteristics. Each attribute is relative to the baseline, which is Male, Half-body, Medium skin color, Non-Human. A positive and significant variable means a preference for that variable. A negative and significant parameter is a negative preference. The mean results show the aggregate effects. The standard deviation results show how heterogeneous the responses are.

Results from the mixed logit model indicate that there were no significant differences in the mean preferences for VCs gender. The standard deviation coefficient for females is significant showing heterogeneous effects for gender. Figure 2 Graph A shows that the mean response is symmetrical and around zero, with about half of participants preferring a female VC (positive coefficient) and half preferring a male VC (negative coefficients); therefore, there is no statistical significance of the mean parameter for gender. With regards to body type torso was less preferred to half body, but full body was preferred to half-body. 
There were also heterogeneous effects for torso. In Figure 2 Graph B the distribution of torso is negative, meaning everybody disliked it. Full bodied VCs had a positive distribution indicating that all participants liked it (Figure 2 Graph C). For style of VC realistic was preferred to non-human while stylized was no different than non-human. Realistic and stylized VCs also had heterogeneous effects. The distribution for realistic VCs shows that although a group of participants disliked them the mass concentration of the distribution was positive (Figure 2 Graph D). Stylized VCs were almost evenly distributed among positive and negative coefficients, meaning some people liked it while others didn't (Figure 2 Graph E).

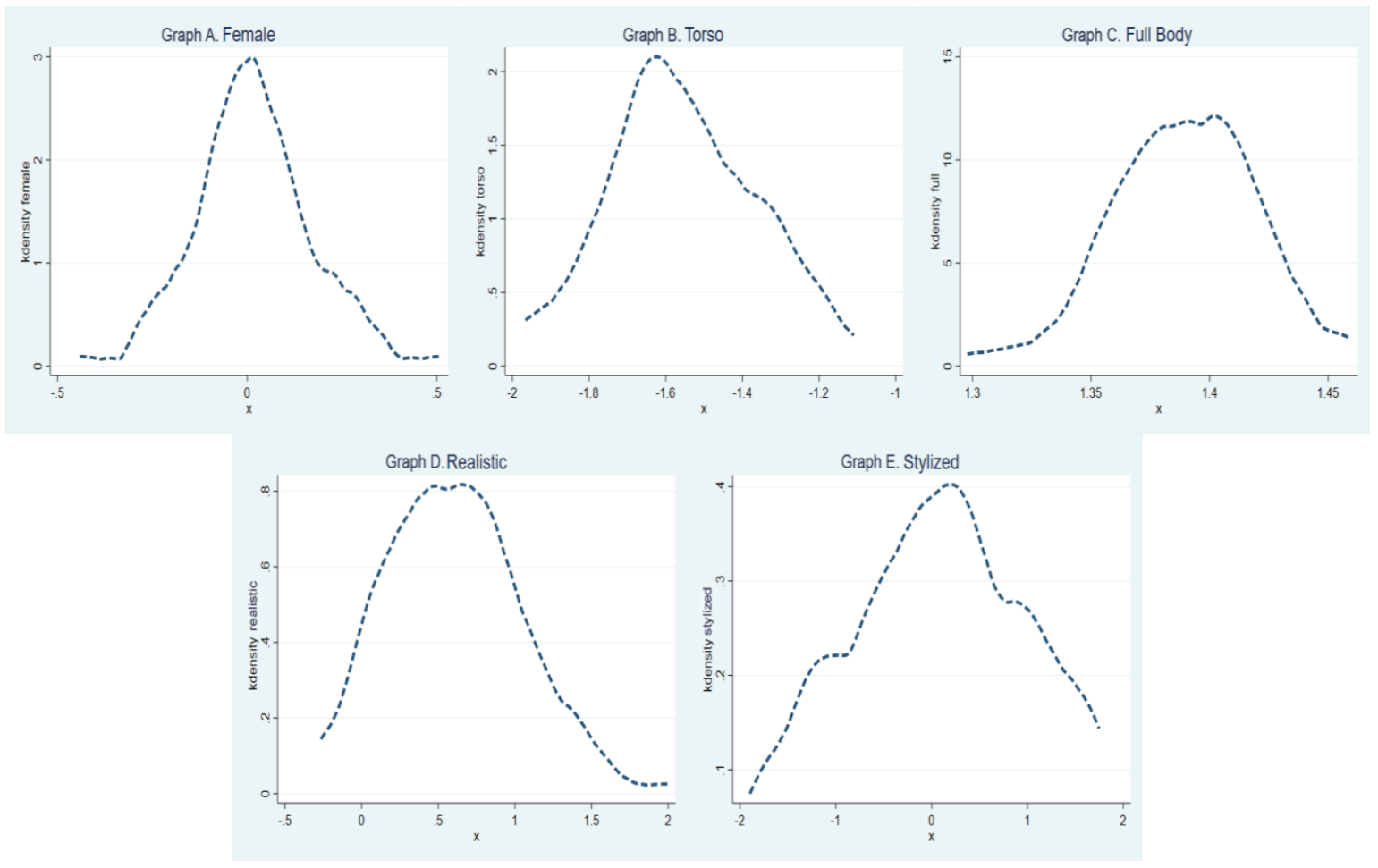

Figure 2. Mixed logit model graphs

\section{DESIGNING THE VR LECTURE APPLICATION}

In this section we discuss shortly the findings from the survey obtaining perceptions of VCs followed by a description of how we designed the VR Lecture application. For VC style we found stylized characters were engaging, familiar, human, and trustworthy, while non-human style characters were more human and familiar. Results of instructor preferences indicated that female was preferred over male, torso is less preferred to half-body while full body is preferred to half body, and realistic VCs were preferred to non-human while stylized VCs were no different than non-human. Based on these results, we designed a lecture VR application with a virtual instructor in a simulated classroom that was female and full bodied. Researchers were interested in comparing realistic VCs to stylized VCs and therefore decided to make both a realistic and stylized VC instructor. In the developed virtual reality application, the instructor stands on a stage behind a lectern, and faces the VR user, who sits in a seat in front of the stage. The instructor then gives a 10-minute psychology lecture, speaking directly to the user while supplementary visuals and text appear on a projection screen (Figure 3 ). 


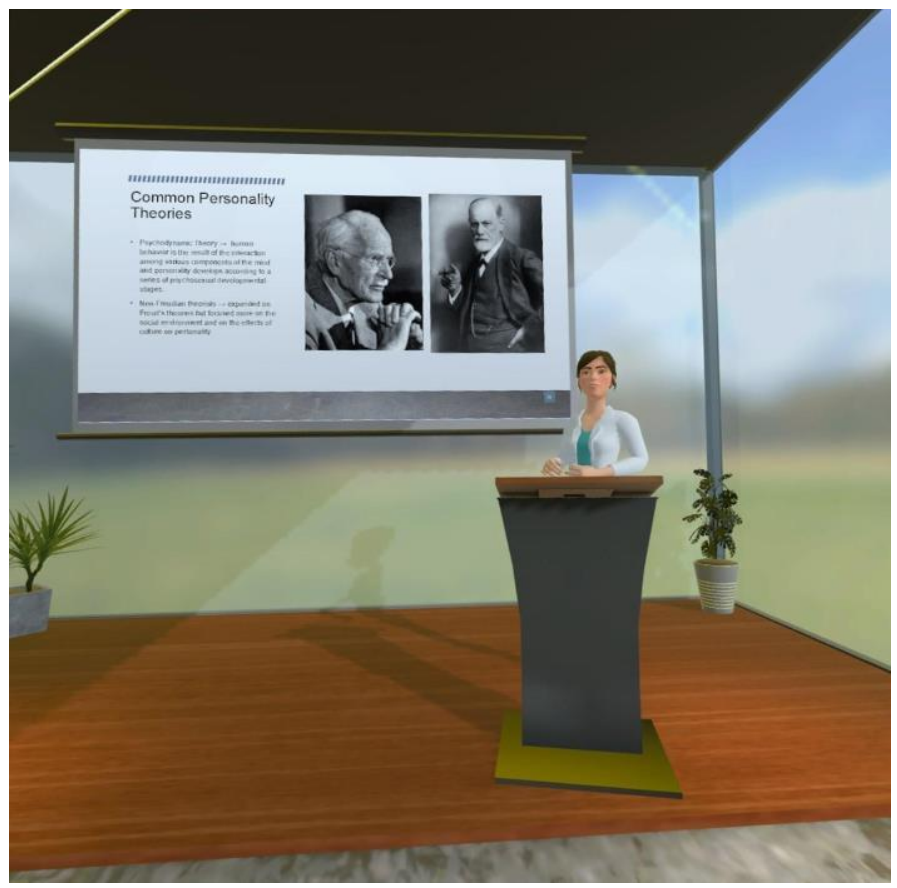

Figure 3. VR lecture user view

\subsection{Design of the Virtual Instruction}

We began the design of our VCs by creating two female personas (Maria and Amanda). We then used a software package, Fuse (Adobe, 2020), to create a photo-realistic character model for each VC based on personas and concept art. Once we had the two realistic models, we were then able to create stylized versions of each. The stylized models were built from an existing base mesh and later modified in a digital sculpting program, Zbrush (Pixologic, 2020). This resulted in four total instructors for our experiment: two realistic characters built in Fuse, and two stylized models based on the realistic characters (Figure 4).

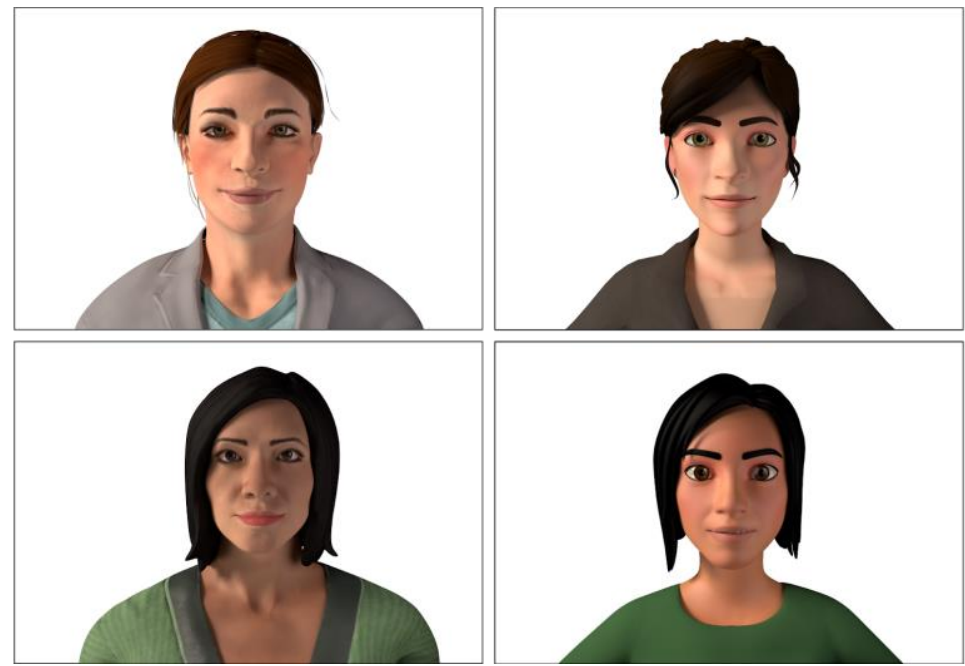

Figure 4. 3D Virtual Instructors: Amanda (Top) and Maria (Bottom) 


\subsubsection{Character Appearance}

Character Stylization: There are numerous ways that a VC can be abstracted or stylized. For this project, we focused primarily on four processes: exaggerate the body's proportions; simplify the shape or profile of features; eliminate non-expressive anatomical details; and create a less-detailed skin texture. In this process of stylizing our realistic VCs many different aspects of their design were impacted. However, specific features like skin color, nose width, jaw size, hair style, and eye shape were preserved to maintain an identifiable relationship between the realistic models and their stylized versions.

Body Shape: We established a design target to use as reference for effective 3D character stylization. We went with female characters from Disney's animated film "Big Hero 6" (Hall and Williams, 2014). Working from screenshots and artwork, the team's 3D artists used Zbrush (Pixologic, 2020) to morph our character's body shapes. For a female character in a modern Disney film, that means the head is larger than real life while the torso, hands, and feet remain small. The silhouette of the body is simplified to resemble more geometric shapes (like triangles and ovals) and lines, markers become streamlined.

Facial Proportion: The face was treated the same way: shapes were simplified, proportions were exaggerated, features that were unique to the character were accentuated. Special attention was given to the most expressive parts of the face: eyes, mouth, and eyebrows. These regions are integral to reading information in the face and are cornerstones for facial recognition algorithms (Sadr, Jarudi, and Sinha, 2003) and taxonomy systems like the Facial Action Coding System (Ekman, Rosenberg, 1997). Large eyes are a common feature in animated films and have the added benefit of making the subject appear more trustworthy (Zebrowitz, Voinescu, and Collins, 1996).

\subsubsection{Character Motion}

We had access to a motion-capture stage with a VICON Motion Capture Unit System. A university professor donned the suit to act out the lecture, movements were later mapped to a 3D skeleton and cleaned in Motionbuilder (Autodesk, 2020). The same motion and skeleton were applied to all four VCs. Mouth animation was generated procedurally with SALSA LipSync (Crazy Minnow Studio, 2019). A script was fed into the software, which translated the text into visemes that matched the words.

\subsubsection{Interactions}

In this version of the application user interactions are minimal. In the application users are greeted with a start menu. After pressing the start button users enter the virtual classroom which consists of four interaction options: pausing and resuming the lesson, a summary of the lesson and the instructor, and an exit option.

\subsubsection{Lesson Development}

The research team decided to cover two topics in psychology: Stress, Lifestyle and Health and Introduction to Personality Psychology. We utilized open educational resources to create initial outlines and created slides covering the two topics, based on which transcripts for the presentation were created. We worked with a professional voice actress to create supplemental audio for the lectures.

\section{VR LECTURE PILOT STUDY}

We conducted a between-subjects pilot study with 12 participants ( 7 male and 5 female) whose ages ranged from 18-24 (5) and 25-34 (7). Participants were recruited through word of mouth. Participants were randomly assigned to two of eight possible conditions which consisted of the learning topic (Personality Psychology or Stress, Health and Wellbeing), VC (Maria or Amanda) and VC style (realistic or stylized), which were then counterbalanced for session order. Participants were greeted by a researcher and then debriefed and given a consent form. During the first session, participants were given a pre-survey addressing demographic questions, experience with VR, experience with VCs in VR, prior knowledge on the learning topics and what style of educational VC they would want (e.g., robot, stylized or realistic) as an instructor. After the pre-survey participants used an Oculus Quest 2 headset to experience the VR Lecture, each lasting 10 minutes. After the lesson the participant was given a post-survey which included a short quiz and Likert scale items addressing overall experience, perception of VCs (e.g., familiarity (Hiroi, Ito, and Nakano, 2009), 
engagement (IJsselsteijn, de Kort, and Poels 2013), trust (Chae, Lee, and Seo, 2016) and humanness (Ho and MacDorman, 2010) and learning experience. Participants were then offered a five-to-ten-minute break if needed, if not the study continued. In the second session participants answered a short pre-survey addressing prior knowledge of the second learning topic and then experienced the second lesson. After completing the session, the participant was given a post-survey which contained the same constructs as the first. Once the post survey was completed a short semi-structured interview was conducted, participants were debriefed and thanked for their time.

\subsection{Results of the VR Lecture Study}

Of these participants 11 had experience with VR using predominantly head mounted displays (HMDs) followed by mobile VR. 6 participants had experience with VCs in VR specifically those that are stylized followed by realistic in gaming applications. When asked what style VC they would like to see in an educational setting 8 participants wanted stylized and 4 wanted realistic VCs. Independent samples t-tests were conducted to see if there were any differences in the perceptions participants had on the VC's style (e.g., realistic or stylized) with regards to familiarity, engagement, trust, humanness, and learning. There were no significant differences found for any of the constructs (Table 1).

Table 1. Independent samples t-test results

\begin{tabular}{lllllll}
\hline Construct & Group & $\mathbf{M}$ & SD & $\boldsymbol{t}$ & df & $\boldsymbol{p}$ \\
\hline Familiarity & Realistic & 4 & .95 & -.516 & 22 & .068 \\
& Stylized & 4.17 & .39 & & & \\
\hline Engagement & Realistic & 3.31 & .96 & .286 & 22 & .917 \\
& Stylized & 3.2 & .95 & & & \\
\hline Trust & Realistic & 3.96 & .75 & .722 & 22 & .935 \\
& Stylized & 3.75 & .66 & & & \\
\hline Humanness & Realistic & 3.38 & .74 & -1.15 & 22 & .115 \\
& Stylized & 3.79 & .52 & & & \\
\hline Learning & Realistic & 3.75 & .62 & -1.149 & 22 & .157 \\
& Stylized & 4 & .43 & & & \\
\hline
\end{tabular}

\section{DISCUSSION AND CONCLUSION}

We investigated how different types of VCs impact users' perception and support learning. Despite there not being any significant differences in t-tests, like the survey results, we found that stylized characters were more familiar and human. This finding is like previous findings in VR research where stylized characters are more appealing than realistic (McDonnell, Breidt, and Bülthoff, 2012). This preference can be related to the uncanny valley where users are comfortable with VCs that appear realistic but still have robotic aspects and tendencies to them. Unlike the survey we found that realistic characters were more engaging and trustworthy. This could be because participants in the survey were looking at a $2 \mathrm{D}$ image of a $3 \mathrm{D}$ character while participants in the pilot study were in an immersed environment and able to see the 3D character in front of them. Another possibility to this finding can be attributed to the behavior of instructing, as previous research has found that behavior of a realistic character impacts user's affinity towards that character (Zibrek, Kokkinara, and McDonnell, 2018). Cui et al (2021) conducted a study investigating the realism of a VC in their Japanese language learning application, Daigaku Life, and found that users were more immersed and found the VC more realistic based on the conversational and instructional behavior exhibited (Cui et al, 2021). With regards to learning, participants felt as if they learned more about the lesson topics with stylized characters, this is interesting because participants rated the realistic style character as being more engaging. This finding aligns with research done by Vicneas and Zamzuri (2019) who found cartoon styled characters resulted in greater perceptions of learning than realistic (Vicneas and Zamzuri, 2019). Implications from this work include information on how to design VC instructors for lecture purposes. By developing trustworthy and engaging VCs we can provide students with a more immersive remote learning experience, which is 
greatly needed in the current times. In future research we aim to design and compare male VCs to the female in a larger study. Limitations of this study include i.) for the survey, participants rated 2D images of 3D VCs without interaction; ii.) for the pilot study there was a small sample size and the users had minimal interaction in the learning environment.

\section{ACKNOWLEDGEMENT}

T3 Grant Program at Texas A\&M University; the Visualization Undergraduate Research Students who participated in this project.

\section{REFERENCES}

Adobe. 2020. Fuse [Computer Software]. Retrieved from https://www.adobe.com/wam/fuse.html.

Agarwala, A. and Lee, J. 2016. Spatial. [online] Available at: https://spatial.io/ (Accessed 11 June 2020).

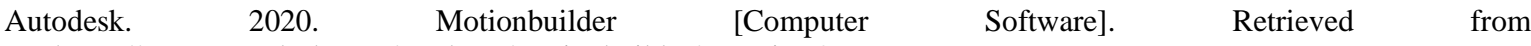
https://www.autodesk.com/products/motionbuilder/overview?.

Chae, S.W., Lee, K.C. and Seo, Y.W., 2016. Exploring the effect of avatar trust on learners' perceived participation intentions in an e-learning environment. International Journal of Human-Computer Interaction, 32(5), pp.373-393.

Crazy Minnow Studio. 2019. SALSA LipSync [Computer Software]. Retrieved from https://crazyminnowstudio.com/unity-3d/lip-sync-salsa/.

Cui, D., Whittinghill, D., Fukada, A., Mousas, C., and Adamo, N., 2021. Interacting with virtual instructors: The effect of gender and years of study on the perception of in-game instructors. Computer Animation and Virtual Worlds., 32(3-4), p.e2026.

Dede, C., 2009. Immersive interfaces for engagement and learning. science, 323(5910), pp.66-69.

Doghead Simulations. 2017. rumii. [online] Available at: https://www.dogheadsimulations.com/rumii (Accessed 9 June 2020).

Ekman, P., and Rosenberg, E.L. eds., 1997. What the face reveals: Basic and applied studies of spontaneous expression using the Facial Action Coding System (FACS). Oxford University Press, USA.

Erolin, C., Reid, L. and McDougall, S., 2019. Using virtual reality to complement and enhance anatomy education. Journal of visual communication in medicine, 42(3), pp.93-101.

Big Hero 6 (2014) Directed by Don Hall and Chris Williams [Film]. Walt Disney Animation.

Hiroi, Y., Ito, A. and Nakano, E., 2009. Evaluation of robot-avatar-based user-familiarity improvement for elderly people. KANSEI Engineering International, 8(1), pp.59-66.

Ho, C.C. and MacDorman, K.F., 2010. Revisiting the uncanny valley theory: Developing and validating an alternative to the Godspeed indices. Computers in Human Behavior, 26(6), pp.1508-1518.

Howland, A. 2012. Virbela. [online] Available at: https://www.virbela.com/ (Accessed 11 June 2020).

HTC Vive. 2018. VIVE Sync. [online] Available at: https://sync.vive.com/ (Accessed 5 June 2020).

IJsselsteijn, W.A., de Kort, Y.A. and Poels, K., 2013. The Game Experience Questionnaire. Eindhoven: Technische Universiteit Eindhoven.

Immersive VR Education Ltd. 2016, March. Engage. [online] Available at: https://engagevr.io/ (Accessed 9 June 2020).

Johnson, W.L., Rickel, J.W. and Lester, J.C., 2000. Animated pedagogical agents: Face-to-face interaction in interactive learning environments. International Journal of Artificial intelligence in education, 11(1), pp.47-78.

Krämer, N.C. and Bente, G., 2010. Personalizing e-learning. The social effects of pedagogical agents. Educational Psychology Review, 22(1), pp.71-87.

Kuhfeld, W. and Cai, W., 2013. Introducing the new ADAPTIVEREG procedure for adaptive regression. In Proceedings of the SAS Global Forum 2013 Conference, Cary, NC: SAS Institute Inc. Available at http:/support. sas. com/resources/papers/proceedings13/457-2013. pdf.

Mayer, R.E. and DaPra, C.S., 2012. An embodiment effect in computer-based learning with animated pedagogical agents. Journal of Experimental Psychology: Applied, 18(3), p.239.

McDonnell, R., Breidt, M. and Bülthoff, H.H., 2012. Render me real? Investigating the effect of render style on the perception of animated virtual humans. ACM Transactions on Graphics (TOG), 31(4), pp.1-11.

Microsoft. 2015, May. AltspaceVR. [online] Available at: https://altvr.com/ (Accessed 9 June 2020). 
Mori, M., MacDorman, K.F. and Kageki, N., 2012. The uncanny valley [from the field]. IEEE Robotics \& Automation Magazine, 19(2), pp.98-100.

Mozilla Mixed Reality Team. 2018. Mozilla Hubs. [online] Available at: https://hubs.mozilla.com/ (Accessed 1 June 2020).

Paiva, A. and Machado, I., 1998, August. Vincent, an autonomous pedagogical agent for on-the-job training. In International Conference on Intelligent Tutoring Systems (pp. 584-593). Springer, Berlin, Heidelberg.

Pixologic. 2020. Zbrush [Computer Software]. Retrieved from https://store.pixologic.com/.

Platt, R. and Tucker, D. n.d. vSpatial. [online] Available at: https://www.vspatial.com/about\#Team (Accessed 5 June 2020).

Pour, P.A., Hussain, M.S., AlZoubi, O., D’Mello, S. and Calvo, R.A., 2010, June. The impact of system feedback on learners' affective and physiological states. In International Conference on Intelligent Tutoring Systems (pp. 264-273). Springer, Berlin, Heidelberg.

Sadr, J., Jarudi, I. and Sinha, P., 2003. The role of eyebrows in face recognition. Perception, 32(3), pp.285-293.

Steptoe, W., Steed, A., Rovira, A. and Rae, J., 2010, April. Lie tracking: social presence, truth and deception in avatar-mediated telecommunication. In Proceedings of the SIGCHI Conference on Human Factors in Computing Systems (pp. 1039-1048).

Sung, Y., Moon, J. and Lin, J.S., 2011. Actual self vs. avatar self: The effect of online social situation on self-expression. Journal of Virtual Worlds Research, 4(1).

Vicneas, M. and Zamzuri, A., 2019. Different Realism Designs of 2D Virtual Agents and its' Arousal Effect on Students' Emotions in Learning.

VRChat Inc. 2014, January. VRChat. [online] Available at: https://hello.vrchat.com/ (Accessed 11 June 2020).

Wagner, D., Billinghurst, M. and Schmalstieg, D., 2006, June. How real should virtual characters be?. In Proceedings of the 2006 ACM SIGCHI international conference on Advances in computer entertainment technology (pp. 57-es).

Yee, N. and Bailenson, J.N., 2006. Walk a mile in digital shoes: The impact of embodied perspective-taking on the reduction of negative stereotyping in immersive virtual environments. Proceedings of PRESENCE, 24, p.26.

Yee, N., Bailenson, J.N. and Rickertsen, K., 2007, April. A meta-analysis of the impact of the inclusion and realism of human-like faces on user experiences in interfaces. In Proceedings of the SIGCHI conference on Human factors in computing systems (pp. 1-10).

Zebrowitz, L.A., Voinescu, L. and Collins, M.A., 1996. " Wide-eyed" and" crooked-faced": Determinants of perceived and real honesty across the life span. Personality and social psychology bulletin, 22(12), pp.1258-1269.

Zibrek, K., Kokkinara, E. and McDonnell, R., 2018. The effect of realistic appearance of virtual characters in immersive environments-does the character's personality play a role?. IEEE transactions on visualization and computer graphics, 24(4), pp.1681-1690. 